\title{
OsNAC45 is Involved in ABA Response and Salt Tolerance in Rice
}

\author{
Xiang Zhang, Yan Long, Jingjing Huang and Jixing Xia ${ }^{*}$ (i)
}

\begin{abstract}
Background: Salt stress threatens crop yields all over the world. Many NAC transcription factors have been reported to be involved in different abiotic stress responses, but it remains unclear how loss of these transcription factors alters the transcriptomes of plants. Previous reports have demonstrated that overexpression of OsNAC45 enhances salt and drought tolerance in rice, and that OsNAC45 may regulate the expression of two specific genes, OSPM1 and OsLEA3-1.

Results: Here, we found that ABA repressed, and $\mathrm{NaCl}$ promoted, the expression of OsNAC45 in roots. Immunostaining showed that OsNAC45 was localized in all root cells and was mainly expressed in the stele. Loss of OsNAC45 decreased the sensitivity of rice plants to ABA and over-expressing this gene had the opposite effect, which demonstrated that OsNAC45 played an important role during ABA signal responses. Knockout of OsNAC45 also resulted in more ROS accumulation in roots and increased sensitivity of rice to salt stress. Transcriptome sequencing assay found that thousands of genes were differently expressed in OsNAC45-knockout plants. Most of the down-regulated genes participated in plant stress responses. Quantitative real time RT-PCR suggested that seven genes may be regulated by OsNAC45 including OsCYP89G1, OsDREB1F, OsEREBP2, OsERF104, OsPM1, OSSAMDC2, and OsSIK1.

Conclusions: These results indicate that OSNAC45 plays vital roles in ABA signal responses and salt tolerance in rice. Further characterization of this gene may help us understand ABA signal pathway and breed rice plants that are more tolerant to salt stress.
\end{abstract}

Keywords: OsNAC45, Rice, Salinity, Salt tolerance, ABA

\section{Background}

Rice is one of the world's most important crop plants and provides daily sustenance for half of the global human population. However, natural stresses including salinity, inappropriate temperatures or drought cause plant growth retardation and thus reduce crop yields (Sachs and Ho 1986; Zhu 2002; VanWallendael et al. 2019). Salt stress affects crops all over the world; high salinity causes osmotic stress and unbalances ionic homeostasis, leading to growth retardation and decreased agricultural productivity (Zhu 2002; Ismail and Horie 2017). To elucidate how plant responds to

\footnotetext{
* Correspondence: xiajx@gxu.edu.cn

State Key Laboratory for Conservation and Utilization of Subtropical Agro-bioresources, College of Life Science and Technology, Guangxi University, Nanning 530004, China
}

\section{SpringerOpen}

(c) The Author(s). 2020 Open Access This article is licensed under a Creative Commons Attribution 4.0 International License, which permits use, sharing, adaptation, distribution and reproduction in any medium or format, as long as you give appropriate credit to the original author(s) and the source, provide a link to the Creative Commons licence, and indicate if changes were made. The images or other third party material in this article are included in the article's Creative Commons licence, unless indicated otherwise in a credit line to the material. If material is not included in the article's Creative Commons licence and your intended use is not permitted by statutory regulation or exceeds the permitted use, you will need to obtain permission directly from the copyright holder. To view a copy of this licence, visit http://creativecommons.org/licenses/by/4.0/.

these abiotic stresses, numerous stress-related genes have been identified and well-studied. Many of these genes are transcription factors - such as, AP2/EREBP (Riechmann and Meyerowitz 1998), DREB (Liu et al. 1998), bZIP (Kim et al. 2004), MYB (Dai et al. 2007) and NAC (Hu et al. 2006) that also participate in plant development, signal transduction and responses to environmental stimuli. Changing the expression levels of individual transcription factors in plants may alter the expression profiles of many downstream genes and, therefore, may affect the plants' tolerance to environmental stresses (Tang et al. 2012; Shim et al. 2018; Yang et al. 2019).

The NAC (NAM, ATAF1/2, CUC2) family is one of the largest families of stress-responsive transcription factors with 117 and 151 predicted members in 
Arabidopsis and rice (Nuruzzaman et al. 2010), respectively. The family appears to be unique to plants (Puranik et al. 2012). The NAC transcription factors have a highly conserved DNA binding domain located at the $\mathrm{N}$-terminal region, while the C-terminal transcriptional activation domains are highly divergent.

The NAM domain was first identified in petunia, and then the ATAF1/2 and CUC2 domains were reported in Arabidopsis (Souer et al. 1996; Aida et al. 1997; Dai et al. 2007). Previous studies have demonstrated that NAC transcription factors participate in organ development. For example, NAM mediates the formation of embryos and flowers in petunia (Souer et al. 1996). Loss of CUC1 and CUC2 causes the cotyledons of Arabidopsis seedlings to curl like a cup (Aida et al. 1997). Another NAC gene from Arabidopsis, AtNAC1, mediates auxin signaling to regulate lateral root growth in seedlings (Xie et al. 2000). Other reports suggest that NAC genes also play vital role in leaf senescence, phytohormone signal transduction and stress responses (Sakuraba et al. 2015; Mao et al. 2017).

ATAF2 directly binds to the promoter region of NIT2 (Nitrilase 2), thereby mediating auxin biosynthesis in Arabidopsis (Huh et al. 2012). Overexpression of OsNAC2 promotes ABA biosynthesis and suppresses ABA catabolic reactions, which increase the ABA content of rice plants (Mao et al. 2017). Overexpressing SNAC1 (Stress-responsive NAC 1) increases the survival rate of transgenic rice during drought or salt stress $(\mathrm{Hu}$ et al. 2006). SNAC2, which was isolated from upland rice IRA109, increases rice resistance to salt or cold stress ( $\mathrm{Hu}$ et al. 2008). PEG, $\mathrm{ABA}, \mathrm{NaCl}$ and $\mathrm{H}_{2} \mathrm{O}_{2}$ have been shown to induce the expression of another NAC transcription factor in rice, ONAC066 (Yuan et al. 2019), which promotes drought and oxidative stress resistance phenotypes.

It is widely accepted that the phytohormone abscisic acid (ABA) plays important roles in plant abiotic stress responses (Zhu 2002). The expression levels of SNAC1, SNAC2/ OsNAC6, OsNAC5, OsNAC10, OsNAC14, ONAC022 and ONAC066 increase under ABA treatment (Hu et al. 2006; Nakashima et al. 2007; Hu et al. 2008; Sperotto et al. 2009; Jeong et al. 2010; Hong et al. 2016; Shim et al. 2018; Yuan et al. 2019). SNAC1, SNAC2, OsNAC5, OsNAC10, OsNAC14 and ONACO66 increase drought tolerance in rice $(\mathrm{Hu}$ et al. 2006; $\mathrm{Hu}$ et al. 2008; Jeong et al. 2010; Jeong et al. 2013; Yuan et al. 2019) and SNAC1, OsNAC5 and OsNAC6 have been shown to enhance the survival of transgenic plants under salt stress ( $\mathrm{Hu}$ et al. 2006; Nakashima et al. 2007; Takasaki et al. 2010).

ABA also negatively regulates seed germination (Nambara and Marion-Poll 2005). Several stress-related
NAC genes including SNAC1, SNAC2, OsNAC52 and ONAC066 have been shown to increase the sensitivity of plants to ABA (Hu et al. 2006; Hu et al. 2008; Gao et al. 2010; Yuan et al. 2019). The expression of RD26, a NAC transcription factor isolated from dehydrated Arabidopsis, is induced by drought and ABA treatment (Fujita et al. 2004). Transgenic Arabidopsis plants overexpressing $R D 26$ were also found to be more sensitive to ABA treatment than WT plants.

Previous studies reported that overexpressing OsNAC45 enhances salt and drought tolerance, and decreases cold tolerance, in rice, and that OsNAC45 may regulate the expression of two genes known as OsPM1 and OsLEA3-1 (Zheng et al. 2009; Yu et al. 2018). However, the phenotypes of OsNAC45-knockout rice under salt and ABA treatment were not investigated. In this paper, we investigated the phenotypes of transgenic rice seedlings that overexpress OsNAC45, or have lost the function of this gene, under $\mathrm{ABA}$ and $\mathrm{NaCl}$ treatment. We also analyzed the transcriptomes of OsNAC45knockout and WT plants under normal growth conditions and high salinity. Our results showed that OsNAC45 positively regulates ABA signal pathway and is required for salt tolerance in rice.

\section{Results \\ Expression and Transcriptional Activation Analysis of OsNAC45}

To determine the tissue specificity of OsNAC45 expression in rice, different tissues (root, stem, leaf, leaf sheath, spike) of WT plants were harvested and the expression of OsNAC45 was detected by quantitative real time RTPCR (qRT-PCR). OsNAC45 was expressed in all the tissues we examined, with the highest level of expression in the roots, and the lowest level of expression in spikes (Fig. 1a).

Since most NAC transcription factors mediated abiotic stress responses, we investigated the expression level of OsNAC45 during salt and ABA treatments. Time gradient experiments showed that the expression level of OsNAC45 increased under salt treatment and reached the maximum level at $6 \mathrm{~h}$ (Fig. 1b). Dose-dependent experiments demonstrated that the expression level of OsNAC45 was positively correlated with the concentration of $\mathrm{NaCl}$ (Fig. 1c). Although the expression level of OsNAC45 decreased under ABA treatment, this effect was not dose-dependent (Fig. 1d).

Next, we fused the coding region of OsNAC45 to the DNA-binding domain of GAL4 (pGBK-OsNAC45) to examine the transcriptional activity of OsNAC45 in yeast. Cells transformed with the pGBK-OsNAC45 vector grew normally on SD/Trp- and SD/Trp-/His-/Ade- plates, while the negative control pGBKT7 was only able to grow on $\mathrm{SD} / \mathrm{Trp}$ - plates (Additional file 1: Fig. S1). These results 

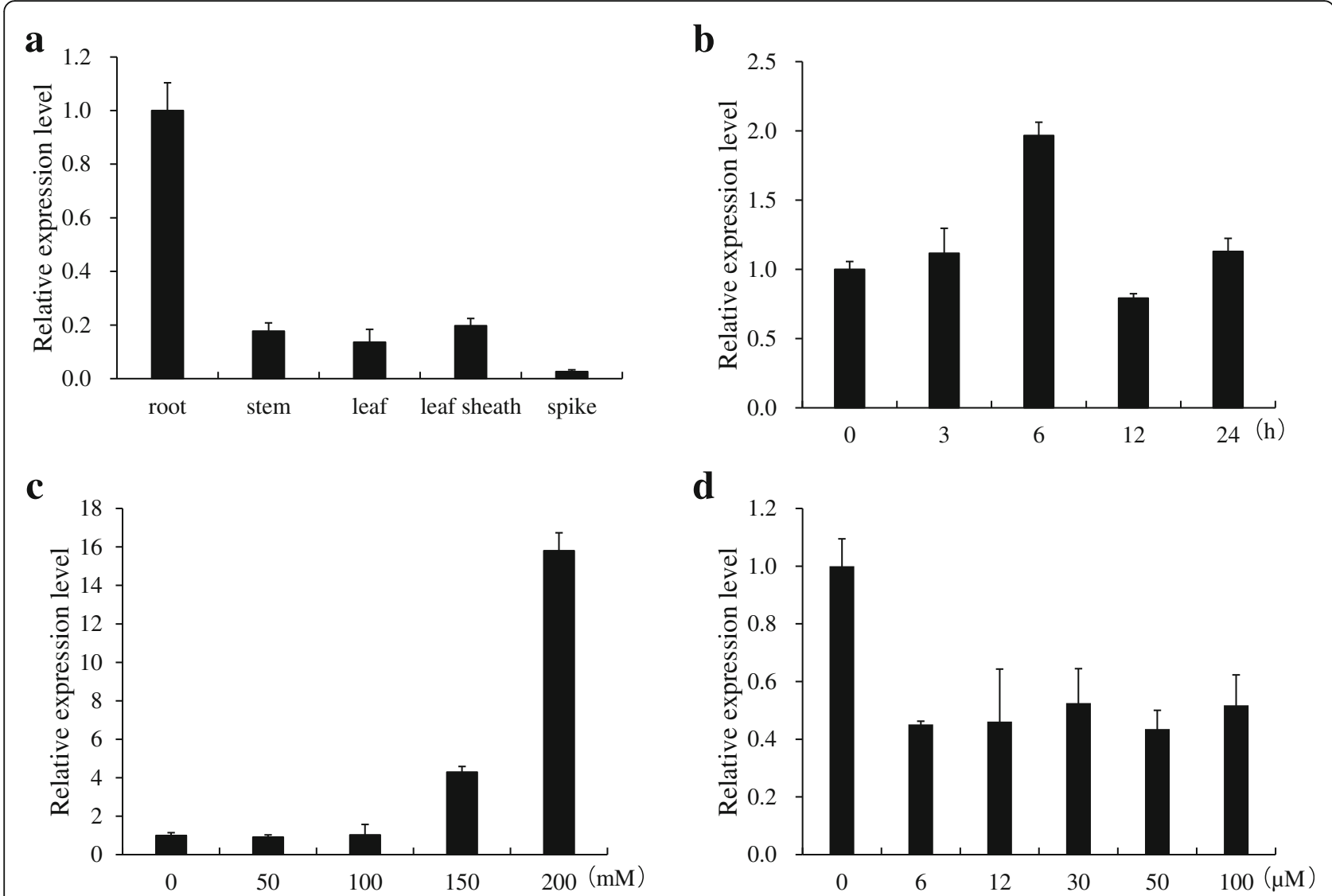

Fig. 1 Expression patterns of OSNAC45. a Expression of OSNAC45 in various tissues of rice cultivar Nipponbare. $\mathbf{b}$ Time-course expression pattern of OsNAC45 in rice roots. Seedlings were exposed to $100 \mathrm{mM} \mathrm{NaCl}$ for 3, 6, 12 or $24 \mathrm{~h}$. c Dose-dependent expression of OsNAC45 in rice roots during salt treatment. Seedlings were exposed to different concentrations of $\mathrm{NaCl}$ for $12 \mathrm{~h}$. $\mathbf{d}$ Dose-dependent expression of OsNAC45 in rice roots during ABA treatment. Seedlings were exposed to different concentrations of ABA for $6 \mathrm{~h}$. Data are means \pm SD of three biological replicates

suggested that OsNAC45 activates transcription of genes in the yeast cells.

\section{Subcellular and Cellular Localization of OsNAC45}

To determine the subcellular localization of OsNAC45 in rice cells, GFP and a OsNAC45-GFP fusion construct were introduced into rice protoplasts with the nuclear marker OsGhd7-RFP. The fluorescence signals of OsNAC45 overlapped nicely with the nuclear marker while fluorescence signals of the control vector (35S: GFP) were distributed in the whole cell (Additional file 1: Fig. S2).

We also examined the cellular localization of OsNAC45 by immunostaining of ProOsNAC45OsNAC45-GFP transgenic rice. DAPI was used as a nuclear stain. Antibodies against GFP (red signal) costained with DAPI, showing that OsNAC45 was localized in nucleus of the rice roots (Fig. 2e-1). This was consistent with our observations in rice protoplast cells. Immunostaining staining also showed that expression level of OsNAC45 was higher in the stele than in other root cell layers (Fig. 2e-h). No signal was observed in the wild-type root (Fig. 2a-d), indicating that antibody against GFP is specific.

\section{ABA Regulates the Germination and Growth of Young OsNAC45-Transgenic Rice Seedlings}

Since exogenous ABA repressed the expression of OsNAC45(Fig. 1d), it is possible that altering OsNAC45 expression in rice may affect the plants' sensitivity to ABA. To confirm this hypothesis, we generated OsNAC45-knockout (osnac45-14, osnac45-15; Additional file 1: Fig. S3) lines using the CRISPR/Cas9 method, and generated OsNAC45overexpression (OE-10, OE-13) lines in which the OsNAC45 gene was driven by the maize ubiquitin promoter (for the expression levels of OsNAC45 in the overexpression lines see Additional file 1: Fig. S4).

First, we compared the ABA sensitivity of the transgenic plants with the WT. In the absence of ABA, the germination rates of the transgenic lines were similar to those of the WT lines (Fig. 3a). However, the seeds of the overexpression lines germinated approximately $12 \mathrm{~h}$ later than the WT seeds (Fig. 3b). After $1 \mu \mathrm{M}$ ABA treatment, the seeds of the osnac45 lines and the WT had nearly all 

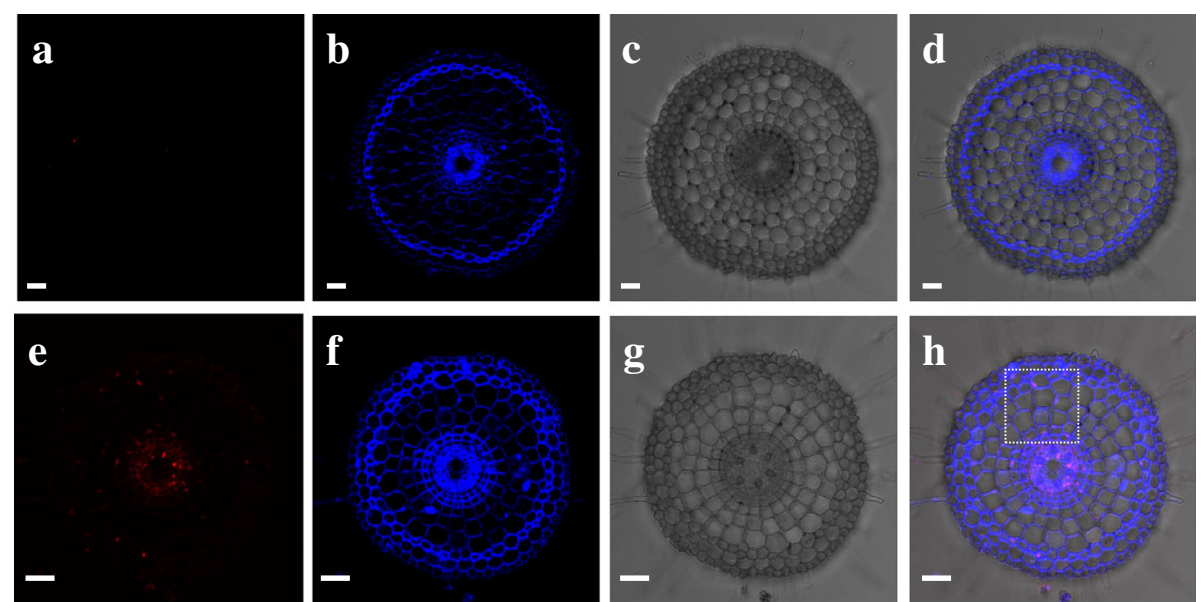

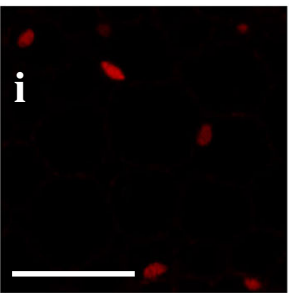

anti-GFP

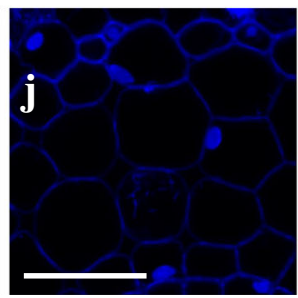

DAPI

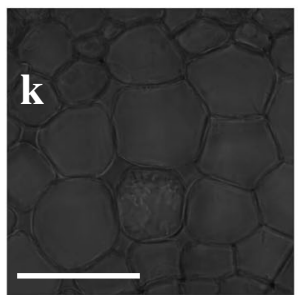

Bright field

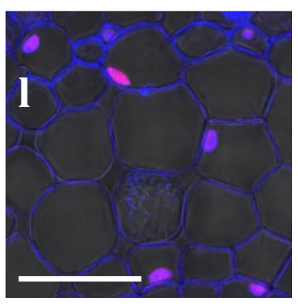

Merged

Fig. 2 Subcellular and cellular localization of OsNAC45. a-d Immunostaining of the roots of Nipponbare using the anti-GFP antibody. e-h Immunostaining of the roots of ProOsNAC45-OSNAC45-GFP transgenic plants. i-I High-magnification images of dotted part in (h). Red color indicates the signal of the anti-GFP antibody, blue color indicates autofluorescence of cell wall and nuclei stained with DAPI. Scale bar $=20 \mu \mathrm{m}$
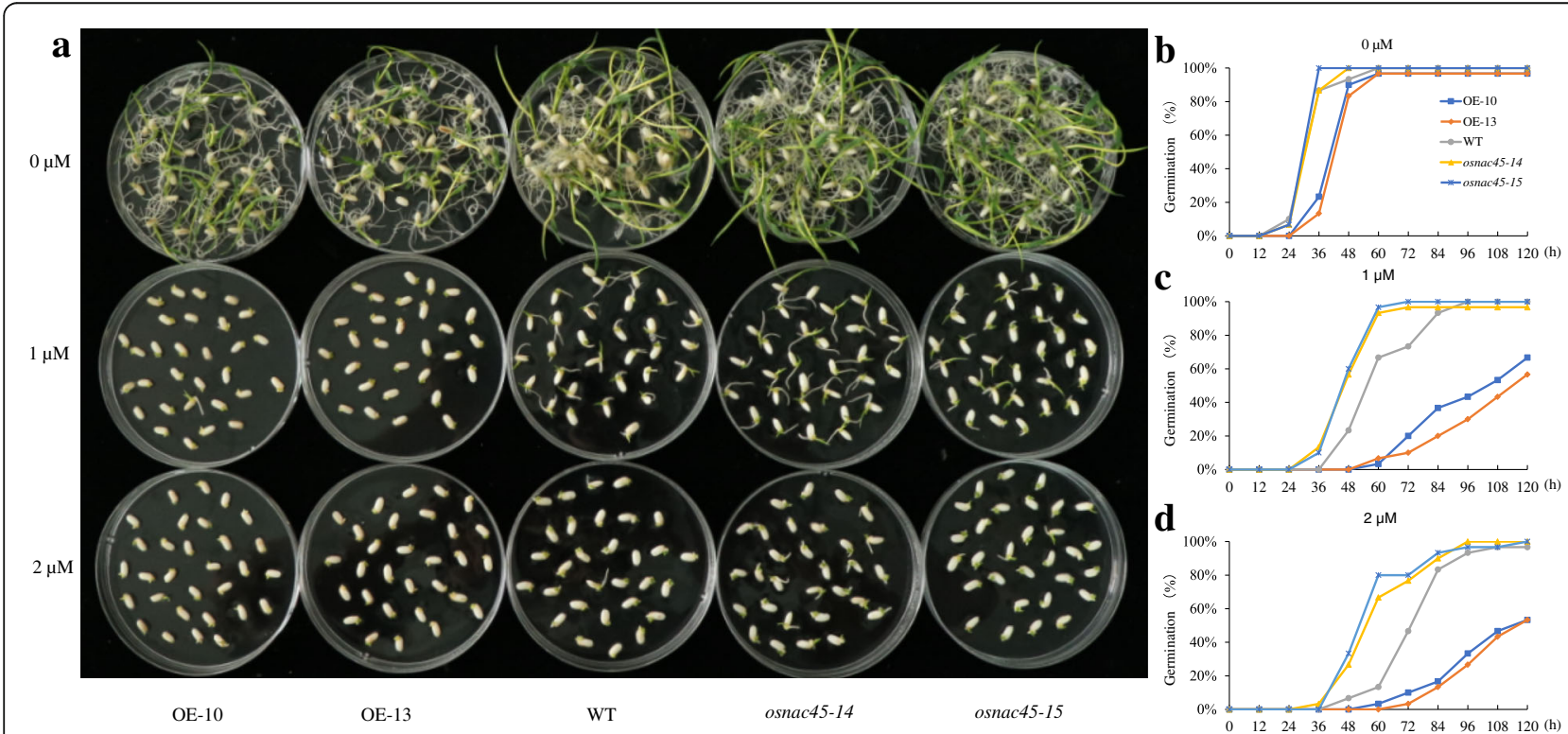

Fig. 3 Germination of OsNAC45-knockout (osnac45-14, osnac45-15) and OsNAC45-overexpression (OE-10, OE-13) lines under different concentrations of ABA. a Germination performance of Nipponbare, osnac45 and OsNAC45-overexpressing seeds after 5 days on 1/2-strength MS medium containing 0,1 , and $2 \mu \mathrm{M}$ ABA. Germination rates of Nipponbare, osnac45 and overexpression seeds on 1/2-strength MS medium containing $0 \mu \mathrm{M}$ ABA (b), $1 \mu \mathrm{M} \mathrm{ABA}(\mathbf{c}), 2 \mu \mathrm{M}$ ABA (d) 
germinated within 72 and $96 \mathrm{~h}$, respectively. However, at $120 \mathrm{~h}$ the germination rates of the overexpression lines had only reached $65 \%$ (Fig. $3 \mathrm{c}$ ). When treated with $2 \mu \mathrm{M}$ exogenous ABA, nearly all the seeds of the osnac45 and WT lines had germinated within 96 and $108 \mathrm{~h}$, respectively. However, only approximately half of the seeds of the overexpression lines had germinated by $120 \mathrm{~h}$ (Fig. 3d).

To further investigate the relationship between OsNAC45 and exogenous ABA, a post-germination assay was performed. Seeds were placed on $1 / 2 \mathrm{MS}$ medium for 2 days to germinate and then seedlings with a similar size were transferred to $1 / 2 \mathrm{MS}$ medium containing different concentrations of ABA. The growth of the osnac45 and OsNAC45-overexpressed lines on the $1 / 2$ MS medium were similar to that of the WT (Fig. 4a, d). In the presence of 1 or $2 \mu \mathrm{M} \mathrm{ABA}$, the shoots of the WT plants were shorter than those of the osnac45 lines, but longer than the shoots of the overexpression lines (Fig. 4b, c, e, f). The results from the germination and post-germination assays suggest that overexpressing OsNAC45 increases the sensitivity of rice plants to ABA treatment and loss of OsNAC45 has the opposite effect.

\section{Loss of OsNAC45 Decreases the Salt Tolerance of Transgenic Rice}

Seedlings of the osnac45 lines and the WT control were exposed to different concentrations of $\mathrm{NaCl}$ to determine their ability to tolerate salt stress. In the absence of $\mathrm{NaCl}$, the growth of the osnac45 lines was similar to that of the WT (Fig. 5a, d). However, when treated with 75 or $100 \mathrm{mM} \mathrm{NaCl}$ for 10 days, the osnac45 lines were smaller and had more withered leaves compared with the WT (Fig. 5b, c). After a 10-day recovery period, the osnac45 lines had fewer green leaves compared with the WT lines (Fig. 5e, f). Under normal conditions, the dry weights of the osnac45 lines and the WT plants were similar (Additional file 1: Fig. S5). After salt treatment the WT plants had greater root and shoot dry weights than the osnac45 lines (Additional file 1: Fig. S5). Furthermore, mineral analysis showed that there was no obvious difference in tissue $\mathrm{Na}^{+}$and $\mathrm{K}^{+}$concentration between WT and osnac45 lines under normal or salt conditions (Additional file 1: Fig. S6). On the other hand, we also compared the accumulation of reactive oxygen species (ROS) in roots between WT and osnac45 lines with the nitroblue tetrazolium (NBT) staining. At the root tip region $(5 \mathrm{~mm})$, the cross section of both WT and osnac45 lines exhibited similar intense staining under normal condition (Fig. 6a-c). Under salt conditions, the staining of osnac45 lines showed more intense while that of WT became weaker compared to the normal conditions, indicating that MT lines accumulated more ROS in roots after salt treatment

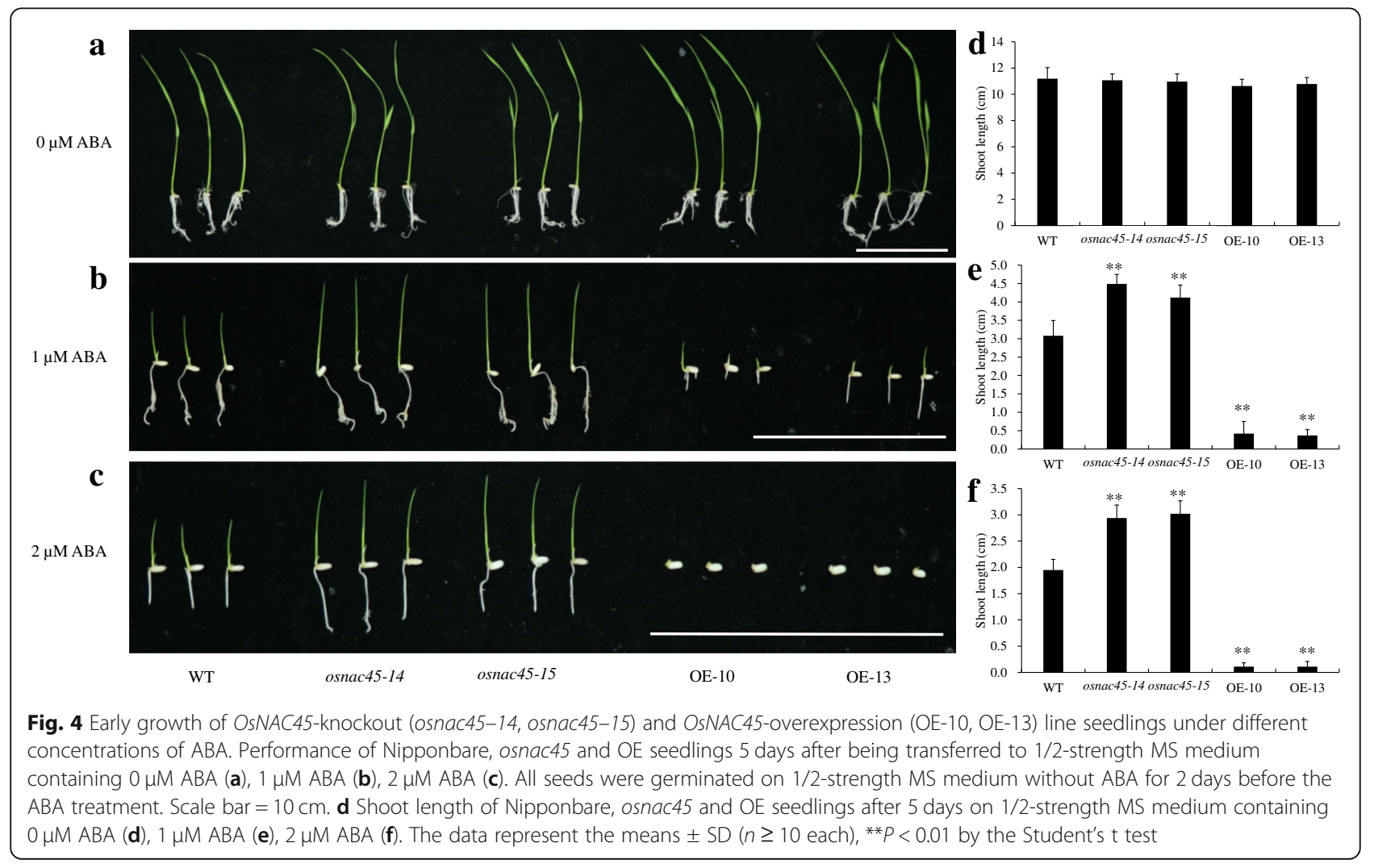



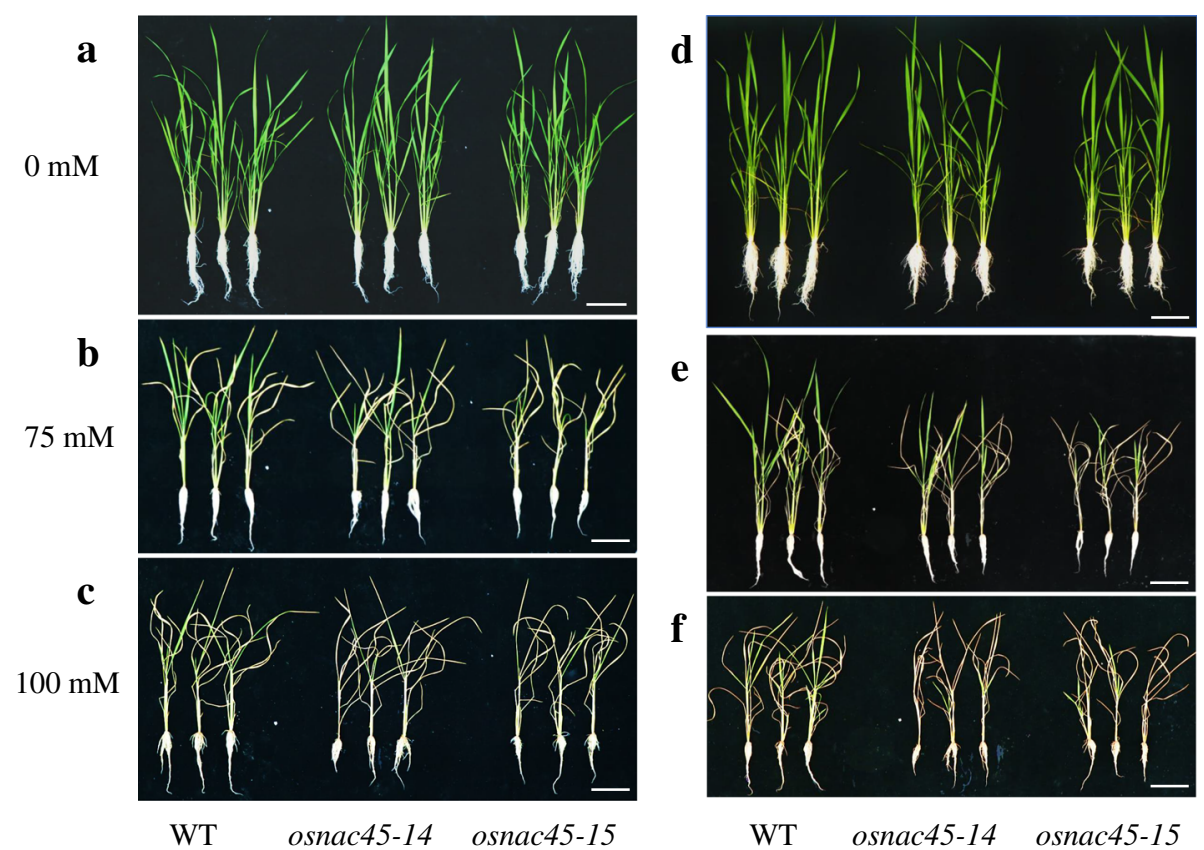

WT osnac45-14 osnac45-15

Treated with $\mathrm{NaCl}$ for 10 days

After 10 days of recovery

Fig. 5 Loss of OsNAC45 decreases salt tolerance in rice. Performance of Nipponbare and osnac45 lines treated with 0 mM (a), 75 mM (b), 100 mM or (c) $\mathrm{NaCl}$ for 10 days. Performance of Nipponbare and osnac45 lines that were allowed to recover for 10 days after the 10-day treatment with 0 $\mathrm{mM}(\mathbf{d}), 75 \mathrm{mM}(\mathbf{e})$ or100 mM (f) NaCl. Scale bar $=10 \mathrm{~cm}$. The experiment was repeated three times and similar results were obtained
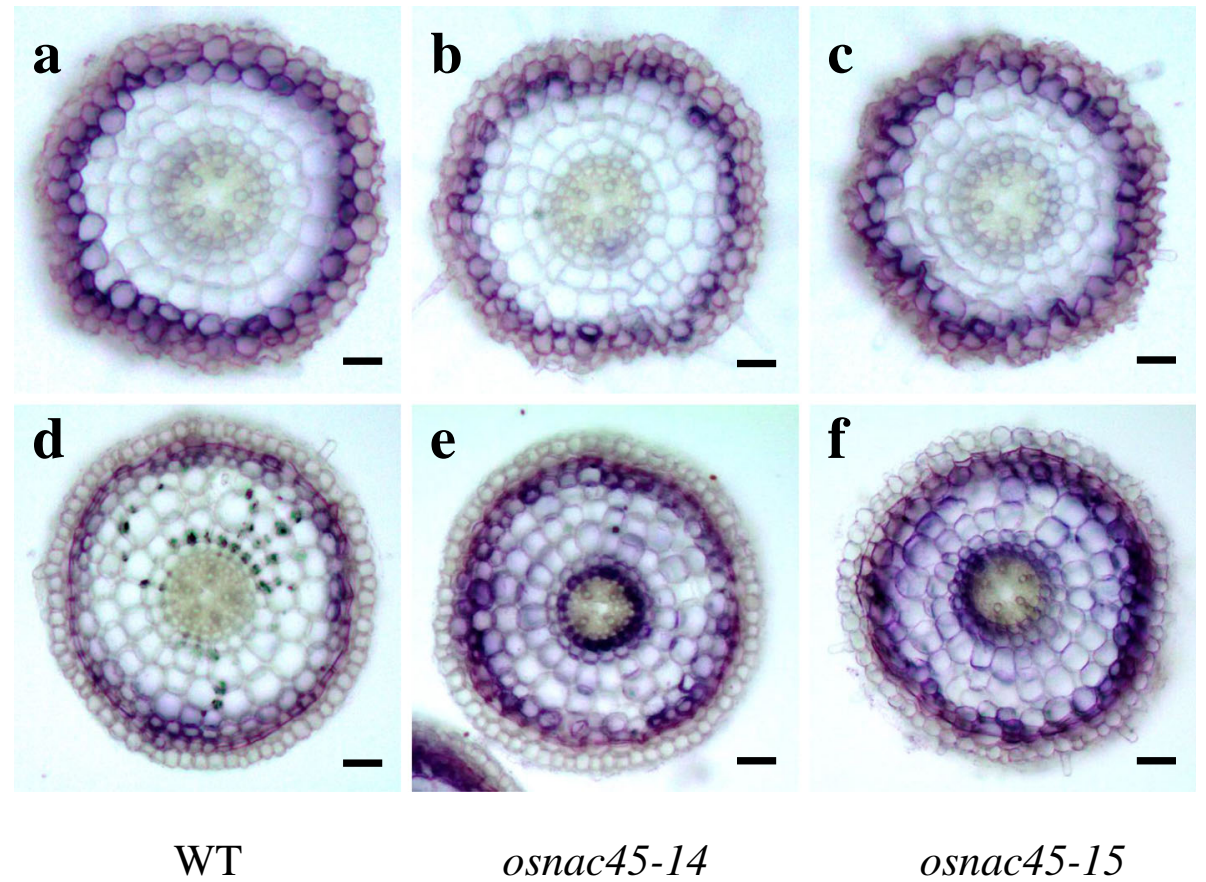

osnac45-15

Fig. 6 NBT staining in the roots of 5-d-old WT and osnac45 seedlings under normal and salt conditions. a-c Cross section of WT, osnac45-14 and osnac45-15 under normal condition, respectively. d-f Cross section of WT, osnac45-14 and osnac45-15 under salt condition, respectively. Seedlings were treated with $100 \mathrm{mM} \mathrm{NaCl}$ for $24 \mathrm{~h}$. Scale bar $=20 \mu \mathrm{m}$ 
(Fig. 6d-f). These results suggest that reduced salt tolerance of osnac 45 lines may be caused by more accumulation of ROS, not $\mathrm{Na}^{+}$and $\mathrm{K}^{+}$in rice.

\section{Loss of OsNAC45 Alters the Expression Profiles of Many Genes in Rice}

To further investigate the role of OsNAC45 in rice under both normal and salt conditions, the transcriptomes of Nipponbare (WT) and mutant osnac45 (MT) rice roots were analyzed using a high-throughput RNA-seq assay. The expression profiles of many genes were significantly different in the MT and WT lines under both normal and salt conditions (Additional file 2). Under normal conditions, 2414 genes were up-regulated and 2746 genes were down-regulated in the MT plants compared with the WT (Additional file 1: Fig. S7a). The upregulated genes are mainly involved in DNA integration, photosystem, photosynthetic membrane, thylakoid components (Fig. 7a). The down-regulated genes are mainly involved in responses to oxidative stress, stress responses, peroxidase activity, oxidoreductase activity and antioxidant activity (Fig. 7b). After treatment with $\mathrm{NaCl}$, a total of 1780 and 1752 genes were up- and down- regulated, respectively, in the MT plants relative to the WT (Additional file 1: Fig. S7b). The GO enrichment of the down-regulated genes in the MT plants indicated that they had decreased stress tolerance, which was consistent with the phenotype of MT under salt stress.

Using a 2-fold change in expression as the threshold, we analyzed the differentially expressed genes (DEGs) between the WT and MT. There were 1408 DEGs between the WT and MT lines under normal conditions, and 1150 DEGs between the lines under salt conditions (Fig. 7c). Among these genes, 162 were continuously upregulated, and 142 were continuously down-regulated, in both conditions (Fig. 7c). We considered these genes to be regulated by OsNAC45. Heat maps show the genes that were differently expressed in the MT and WT lines after salt treatment, which may also be regulated by OsNAC45 (Additional file 1: Fig. S8a, b).

To confirm these results, we performed qRT-PCR to check the expression profiles of some DEGs under both normal and salt conditions. As expected, we found that the changes in the expression levels of the DEGs under different conditions were not the same in the WT and MT plants. After salt treatment, the expression level of six genes was found to be lower in the MT lines than in WT plants including OsCYP89G1 (Os07g0451300), OsDREB1F (Os01g0968800), OsEREBP2 (Os01g0868000), OsERF104 (Os08g0474000), OsSAMDC2 (Os02g0611200), and OsSIK1 (Os06g0130100) (Fig. 8a-f). Except for OsERF104 and OsCYP89G1, other four genes have been reported to be involved in rice salt tolerance (Wang et al. 2008;
Ouyang et al. 2010; Serra et al. 2013; Chen et al. 2014). These results indicate that OsNAC45 probably affected these salt-tolerance related genes to regulate salt tolerance in rice.

Since previous studies hypothesized that OsPM1 (Os05g0381400) and OsLEA3-1 (Os05g0542500) may be regulated by OsNAC45 (Zheng et al. 2009), we performed qRT-PCR to check the expression profiles of these two genes. The results showed that salt treatment induced the expression of OsPM1 by 8 -fold in WT plants, but only by 4 -fold in MT plants (Fig. 8g). However, there were no obvious differences in the expression profiles of OsLEA3-1 in WT and MT plants under normal or salt conditions (Fig. 8h). These results suggest that OsPM1, but not OsLEA3-1, may be regulated by OsNAC45.

\section{Discussion}

In this study, we found that $\mathrm{ABA}$ treatment decreased the expression of OsNAC45, but this effect was not dose-dependent (Fig. 1d). In the absence of ABA, the germination rates of the WT, osnac45 and OsNAC45overexpressed lines were similar, but the seeds of the overexpression lines germinated more slowly (Fig. 3b). A recent study demonstrated that overexpressing OsNAC2 in transgenic rice enhances plant resistance to drought and salt stresses but causes a delay in seed germination (Jiang et al. 2019). This suggests that OsNAC45 and OsNAC2 use similar pathways in ABA signal transduction. After germinating, the shoots of the overexpression lines grew similarly to the WT plants (Fig. 4a, d). In the presence of $\mathrm{ABA}$, the germination and growth of the OsNAC45 overexpression seedlings were severely inhibited compared with the WT (Figs. 3a, c, d; 4b, c, e, f). On the contrary, the osnac 45 seedlings germinated more quickly and grew faster than the WT (Figs. 3a, c, d; 4b, c, e, f). These results demonstrate that OsNAC45 participates in ABA signaling pathways during the germination and early growth of rice.

Gene redundancy sometimes brings obstacles in biological studies: knock-outs or knock-downs of certain genes sometimes fail to provide informative phenotypes that may provide clue to the gene's role. For example, mutant rice plants lacking OsbZIP62 have no obvious mutant phenotypes in response to $\mathrm{ABA}$ treatment, whereas plants overexpressing this gene are hypersensitive to ABA (Yang et al. 2019). A previous study suggested that over-expressing OsNAC45 enhances rice resistance to salt stress (Zheng et al. 2009). To further elucidate the role of OsNAC45 in rice, we used a CRIS PR/Cas9 approach to generate OsNAC45 knock-out lines. We found that the leaves of the osnac45 lines were more withered and yellow compared with the WT after $75 \mathrm{mM}$ or $100 \mathrm{mM} \mathrm{NaCl}$ (Fig. 5b, c). After 10 days of 


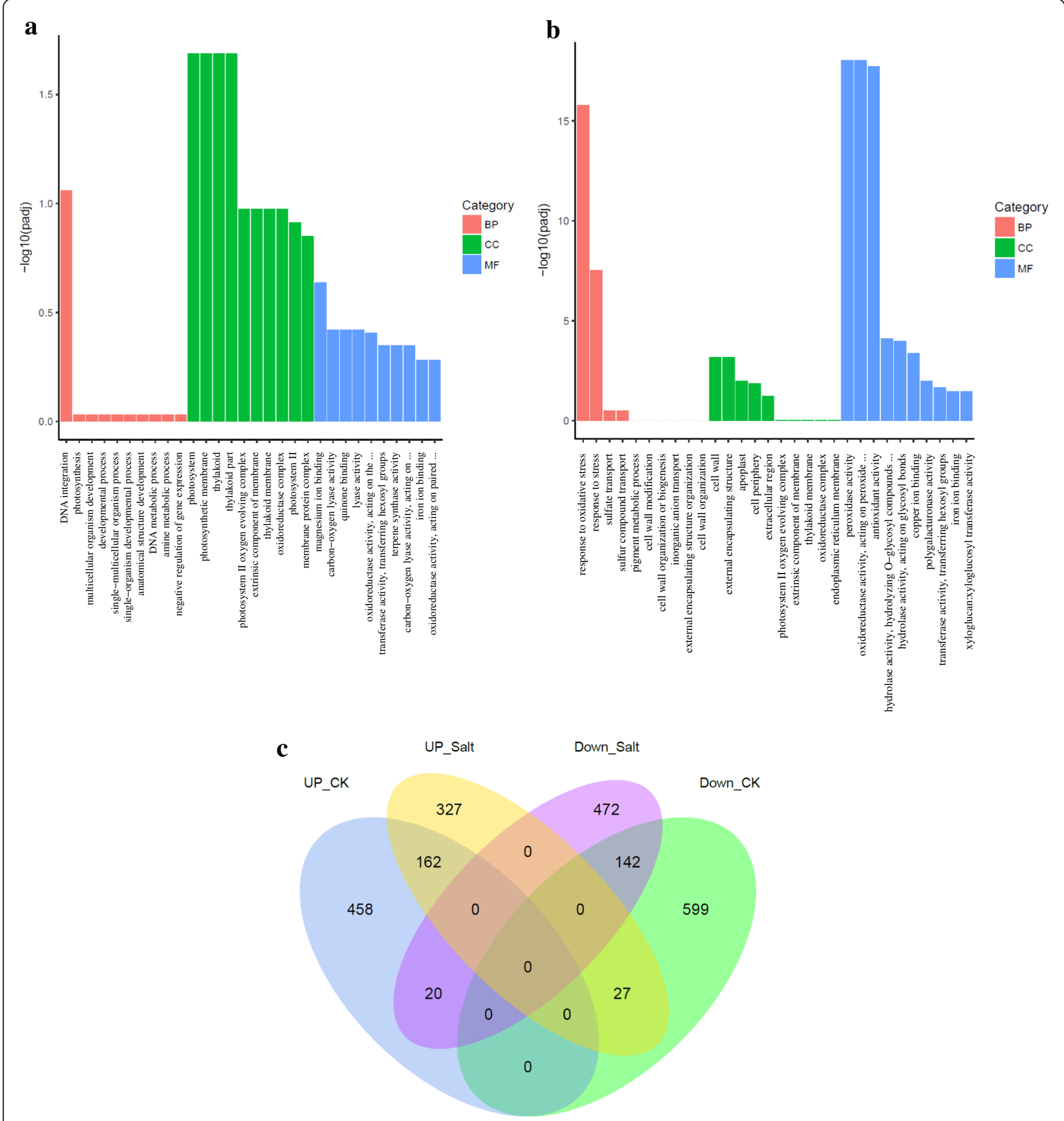

Fig. 7 Transcriptome analysis of OsNAC45-regulated genes. Classification of up-regulated genes (a) and down-regulated genes (b) in osnac45 (MT) transgenic plants compared with the WT under normal conditions. c Venn diagram of the numbers of genes that are regulated by OsNAC45 in the MT and WT lines. UP_CK: the up-regulated genes in the MT compared with the WT under normal conditions (fold-change > 2). UP_SALT: the up-regulated genes in the MT compared with the WT in high salinity (fold-change $>2$ ). DOWN_SALT: the down-regulated genes in the MT with the WT in high salinity (fold-change < 0.5). DOWN_CK: the down-regulated genes in the MT compared with the WT under normal conditions (fold-change < 0.5). BP: biological process. CC: cellular component. MF: molecular function. Three biological replicates $(n=3)$ were performed for each treatment 


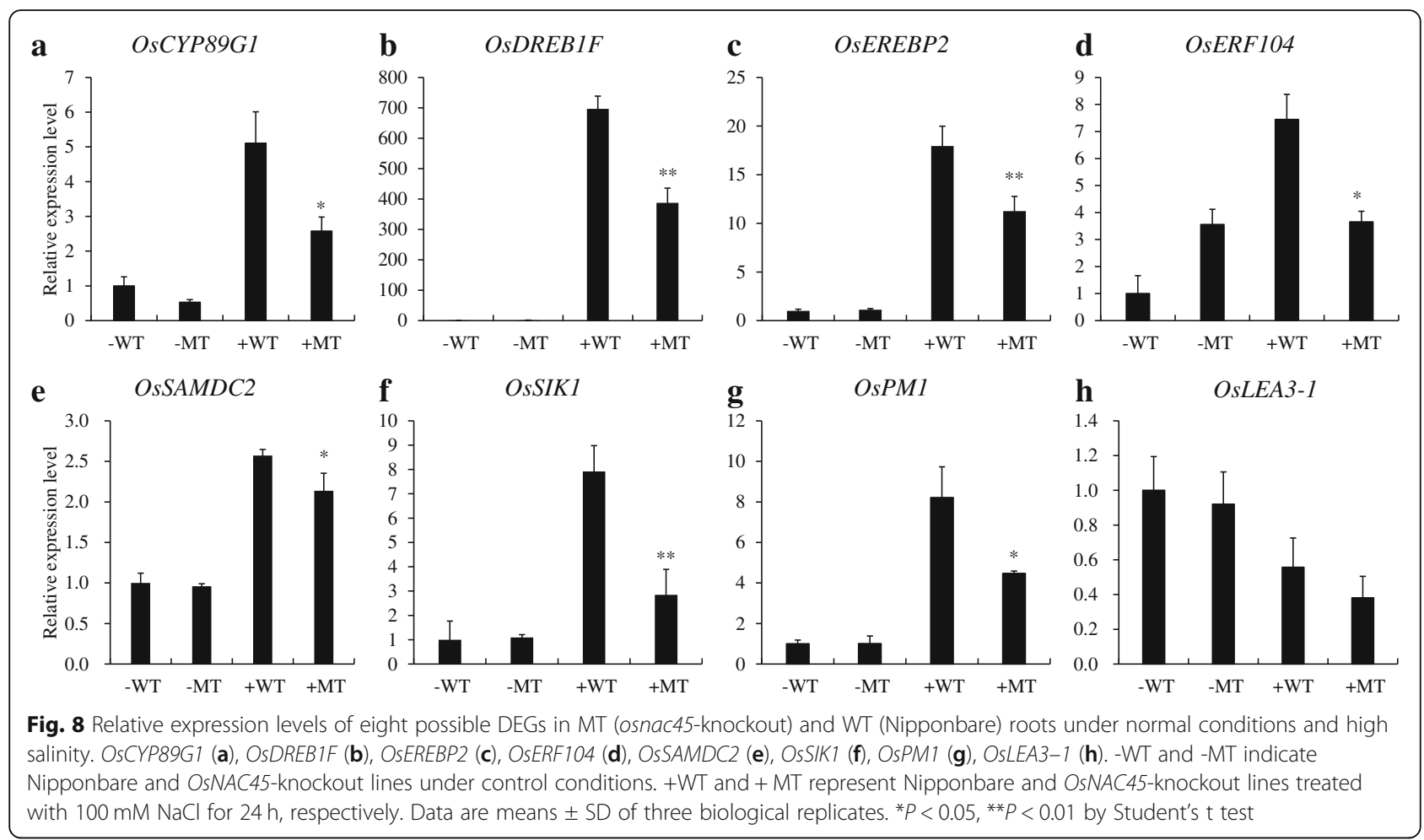

recovery, salt toxicity symptoms were still more noticeable in the osnac45 lines (Fig. 5e, f). The differences in the root and shoot dry weights of WT and MT lines after the salt treatment and recovery period were consistent with their phenotypes observed (Additional file 1 Fig. S5). These results demonstrate that loss of OsNAC45 makes rice more sensitive to salt stress, indicating that OsNAC45 is required for salt tolerance in rice.

To find out how changing the expression of OsNAC45 affects the expression of its downstream genes, we performed an RNA-seq assay. The results showed that the expression levels of over 2000 genes were changed in MT lines under two different conditions (Fig. 7c). Under normal conditions, 2746 genes were down-regulated and gene ontology (GO) annotation analysis showed that these genes mainly belong to following categories: responses to oxidative stress, responses to stress, peroxidase activity, oxidoreductase activity and antioxidant activity (Fig. 7b). These results show that loss of OsNAC45 down-regulates many stress-related genes, which may account for the increased sensitivity of the osnac45 lines to salt stress. Among these downregulated genes, we confirmed the expression levels of six of the down-regulated genes, OsCYP89G1, OsDREB1F, OsEREBP2, OsERF104, OsSAMDC2, and OsSIK1, by qRT-PCR. Previous reports found that OsCYP89G1 and OsERF104 belong to the ERF and CPY families, respectively, and may participate in stress responses, but their exact roles in response to salt stress remain unclear (Mishra et al. 2013; Wei and Chen 2018). OsDREB1F encoding a transcription factor was shown to enhance rice salt tolerance through activating the expression of some stressrelated genes by specifically binding to DRE/CRT element (G/ACCGAC) (Wang et al. 2008). OsEREBP2 was also a transcription factor and mediated rice salt tolerance through regulating a receptor-like kinase OsRMC which is a negative regulator of salt stress response (Serra et al. 2013). OSSAMDC2 and OsSIK1 encoded a S-adenosylmethionine decarboxylase gene and a stress-induced protein kinase gene, respectively. These two genes were proposed to be involved in rice salt tolerance through ROS scavenging pathway. Overexpression of OsSIK1 could efficiently activate antioxidative system (Ouyang et al. 2010). Down-regulation of OsSAMDC2 resulted in reduced many antioxidant enzyme activities (Chen et al. 2014). Therefore, more ROS accumulation in MT roots after salt treatment could be attributed to lower expression level of OsSAMDC2 and OsSIK1 compared with WT.

In a previous study, the expression levels of OsPM1 (plasma membrane protein 1) and OsLEA3-1 (late embryogenesis abundant) were found to be higher in transgenic rice plants overexpressing OsNAC45 than in the WT (Zheng et al. 2009). However, when we measured the expression levels of these two genes in the MT and WT lines, our results indicated that OsPM1, but not OsLEA3-1, may be regulated by OsNAC45 (Fig. 8g, 
h). These findings suggest that OsLEA3-1 expression may be controlled by some other genes that are up-regulated by OsNAC45, but not OsNAC45 itself.

Previous study has reported that over-expressing OsNAC5 and OsNAC6 in rice significantly improves plant salt stress resistance and that OsNAC5 and OsNAC6 regulate the expression of OsLEA3-1 (Takasaki et al. 2010). This suggests that OsNAC45 is involved in different regulatory pathways to OsNAC5 or OsNAC6. OsPM1 encodes a plasma membrane protein that mediates ABA influx through the plasma membrane (Yao et al. 2018). Additionally, OsNAC45 also regulates the expression of OsDREB1F (Fig. 8b), which is reported to be involved in ABA-dependent signal pathway (Wang et al. 2008). These may partially explain the phenotypes we observed in OsNAC45 transgenic rice under ABA treatment.

\section{Conclusions}

In conclusion, our results demonstrate that OsNAC45 plays important roles in $\mathrm{ABA}$ signal responses and is required for rice salt tolerance through regulating the expression of multiple stress-related genes.

\section{Methods}

\section{Generation of Transgenic Plants}

The CRISPR/Cas9 gene editing system was used according to the previously described protocol to generate transgenic OsNAC45 knockout rice plants (Ma et al. 2015). To create a construct to overexpress OsNAC45 in rice (named OsNAC45-OE), cDNA obtained from reverse transcription-PCR (RT-PCR) of Nipponbare total RNA was used as a template to obtain the coding region of OsNAC45. The full-length cDNA of OsNAC45 was inserted into the pCAMBIA1300-Ubi vector carrying the maize Ubiquitin promoter and the terminator of the nopaline synthase gene. OsNAC45-OE and OsNAC45-knockout vectors were then introduced to Agrobacterium tumefaciens strain EHA101 and transformed into wild-type (WT) rice (Oryza sativa cv. Nipponbare). The primers used in this study were listed in Additional file 1 Table S1.

To detect the presence of the desired mutations in the rice plants, we performed DNA sequencing using OsNAC45-specific primers and the sequence chromatograms were aligned with the WT (Oryza sativa cv. Nipponbare) controls. Two homozygous mutants and two overexpressing OsNAC45 lines were selected for further experiments.

\section{Plant Materials and Growth Conditions}

The WT rice (Oryza sativa cv. Nipponbare) was used as a transformation recipient in this study. Rice seeds were soaked in water for 2 days and then the germinated seeds were put on a net floating on $0.5 \mathrm{mM} \mathrm{CaCl} 2$ solution in a greenhouse and used for various experiments.

For tissue specificity analysis, samples of root, stem, leaf, leaf sheath and spike were harvested from Nipponbare plants after the heading stage. For the salt treatment, 7-day-old seedlings of Nipponbare were exposed to different concentrations of $\mathrm{NaCl}(0,50,100$, 150 and $200 \mathrm{mM}$ ) for $12 \mathrm{~h}$ or $100 \mathrm{mM} \mathrm{NaCl}$ for different times $(3,6,12$ and $24 \mathrm{~h})$. For each condition, roots from 5 to 7 plants were collected at every timepoint for RNA extraction. For the ABA treatment, 7-day-old Nipponbare seedlings were exposed to different concentrations of $\mathrm{ABA}(0,6,12,30,50$ and $100 \mu \mathrm{M})$ for $6 \mathrm{~h}$. For each condition, roots of 5-7 plants were sampled at every timepoint for RNA extraction.

\section{RNA Isolation and Gene Expression Analysis}

Rice RNA was extracted with the Trizol reagent kit (Life technologies, USA) according to the manufacturer's instructions. First-strand cDNA was synthesized using a Hiscript II Q RT SuperMix Kit (Vazyme) with $1 \mu \mathrm{g}$ total RNA. Quantitative reverse transcription-PCR (qRTPCR) was performed with ChanQTM SYBR Color qPCR Master Mix (Vazyme) on a StepOnePlus Real-Time PCR System (Analytik Jena AG). Histone H3 was used as an internal standard. The relative expression levels of the genes were calculated by the $2^{-\Delta \Delta C \mathrm{~T}}$ method.

\section{Transactivation Activity Assay}

The full-length coding region of OsNAC45 was amplified by PCR and fused with the GAL4 DNA-binding domain in a pGBKT7 vector. AH109 yeast cells transformed with GBK-OsNAC45 or a control construct were spotted on SD/Trp- and SD/Trp-/His-/Ade- medium and incubated at $30^{\circ} \mathrm{C}$.

\section{Subcellular Localization of OsNAC45}

The subcellular localization of OsNAC45 was investigated in rice protoplasts by fusing OsNAC45 to green fluorescent protein (GFP). Full-length cDNA of OsNAC45 was cloned into the pYL322-GFP vector after the GFP coding region to make the GFP-OsNAC45 vector.

A nucleus marker (GFP-OsGhd7) plus GFP-OsNAC45 or the control pYL322-GFP vector were transformed into rice protoplasts with PEG as previously described (Chen et al. 2011). The protoplasts were placed under a confocal laser scanning microscope to gather images of the GFP fluorescence (TCS SP8; Leica).

\section{Cellular Localization of OsNAC45}

The promoter (2157 bp) of OsNAC45 was amplified by PCR from Nipponbare genomic DNA, and the coding 
region of OsNAC45 without the stop codon was amplified by PCR from cDNA extracted from Nipponbare roots. To make a construct encoding the OsNAC45 protein fused to GFP at its N-terminal end, the amplified fragment was cloned into the pCAMBIA1300-GFP vector. The resulting construct (named ProOsNAC45-OsNAC45-GFP) was transformed into Nipponbare, producing transgenic lines carrying ProOsNAC45-OsNAC45-GFP.

To investigate the cellular localization of OsNAC45, immunostaining was performed using an antibody against GFP as previously described (Yamaji and Ma 2007). Briefly, the roots of WT and transgenic lines carrying ProOsNAC45-OsNAC45-GFP were embedded in $5 \%$ agar and sectioned $100-\mu \mathrm{m}$ thick with a microslicer (VT1000 S, Leica). Sections were placed on microscope slides and incubated with the rabbit anti-GFP polyclonal antibodies and secondary antibodies (Alexa Fluor 555 goat anti-rabbit IgG; Molecular Probes) at room temperature, respectively. Then sections were stained with DAPI. A confocal laser scanning microscope was used to collect fluorescence images (TCS SP8; Leica, wetzlar, Germany).

\section{Germination Assay and Young Seedling Growth Assay}

For the germination assays, rice seeds (30 seeds for each line) were placed on $1 / 2$ MS medium contain 0,1 , and $2 \mu \mathrm{M}$ ABA (purity> $99 \%$, Biotopped) at $28^{\circ} \mathrm{C}$, and the number of germinated seeds were counted every $12 \mathrm{~h}$ for 5 days.

For the young seedling growth assay, rice seeds (30 seeds for each line) were placed on 1/2 MS medium at $28^{\circ} \mathrm{C}$ for 2 days, and plants of a similar size were transferred to a plastic box $(15 \times 11 \times 6 \mathrm{~cm})$ containing $1 / 2$ MS medium containing 0,1 or $2 \mu \mathrm{M}$ ABA. Shoot length was measured 5 days after the transfer.

\section{Determination of Salt Tolerance and Element Concentration}

We exposed 30-day-old seedlings of WT (Nipponbare) and OsNAC45-knockout lines (osnac45-14, osnac45-15) to 0,75 or $100 \mathrm{mM} \mathrm{NaCl}$ for 10 days and then returned the plants to their normal growth conditions for 10 days. After the treatment and recovery time, roots and shoots of the WT and osnac45 were sampled and dehydrated at $70{ }^{\circ} \mathrm{C}$ for 3 days and then the dry weights of the samples were measured. The dried samples were digested in boiling tubes with $65 \% \mathrm{HNO}_{3}$. The $\mathrm{Na}^{+}$and $\mathrm{K}^{+}$concentrations in the digested solution were determined by ICPMS (Plasma Quant MS; Analytik Jena AG).

\section{NBT Staining}

We exposed 5-day-old seedlings of WT (Nipponbare) and OsNAC45-knockout lines (osnac45-14, osnac45-15) to 0 or $100 \mathrm{mM} \mathrm{NaCl}$ for $24 \mathrm{~h}$. The roots of all lines were incubated with $6 \mathrm{mM}$ NBT for $10 \mathrm{~min}$. Then roots were embedded in low melting agarose and sectioned at $5 \mathrm{~mm}$ from root tip (100- $\mu \mathrm{m}$ thickness) using a slicer (VT1000 S, Leica). Sections were photographed by a Nikon E100 microscope.

\section{RNA-seq Experiments}

Two-week-old seedlings of Nipponbare and the OsNAC45 mutant lines (MT) were exposed to $100 \mathrm{mM}$ $\mathrm{NaCl}$ for $24 \mathrm{~h}$ and then the roots of seedlings were sampled for further experiments. Total RNA was extracted and purified to obtain the total mRNA. Then, we synthesized the first strand cDNA and second strand cDNA using the reverse transcriptase and DNA polymerase enzymes, respectively. After constructing the cDNA ibrary, RNA-sequencing was carried out on an Illumina NovaSeq platform. Deseq2 was used to analyze the differences in gene expression between the WT and MT lines. The DEGs (differentially expressed genes) were selected by the standard of $\mid \log 2$ ratio $\mid>2$. GO (Gene Ontology; http://geneontology.org/) analysis of DEGs was carried out by hypergeometric tests, and each $p$-value indicates the enrichment of the corresponding category.

\section{Supplementary Information}

The online version contains supplementary material available at https://doi. org/10.1186/s12284-020-00440-1.

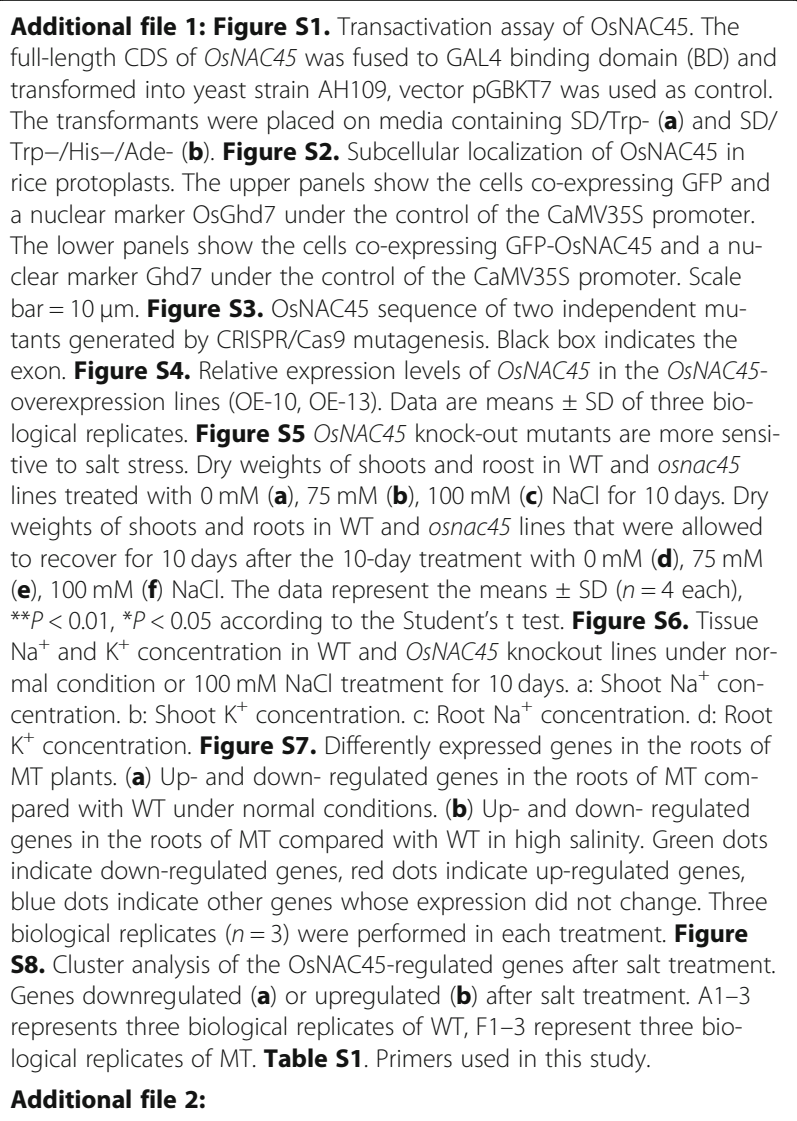




\section{Abbreviations}

ABA: Abscisic acid; NAC: NAM, AFAT, and CUC transcription factor; qRTPCR: Reverse transcription quantitative PCR; WT: Wild-type; MT: Mutant; OE: Overexpression; GFP: Green Fluorescent Protein; ROS: Reactive oxygen species; NBT: Nitroblue tetrazolium

\section{Acknowledgements}

We are very grateful to Prof. Yaoguang Liu from South China Agricultural University for kindly providing us with the pYLCRISPR/Cas9Pubi vector and the pYL322-d1-eGFP vector.

\section{Authors' Contributions}

JX conceived the study and designed the experiments. XZ performed most of the experiments. YL and JH participated in the research. XZ and JX wrote the manuscript. All authors read and approved the final manuscript.

\section{Funding}

The research was funded by National Natural Science Foundation of China (31670253), Guangxi Natural Science Foundation (2016GXNSFFA380013), Hundred-Talent Program of Guangxi (2014), and Guangxi innovation-driven development special funding project (Grant no. Guike-AA17204070).

\section{Availability of Data and Materials}

All data supporting the conclusions of this article are provided within the article (and its additional files).

\section{Ethics Approval and Consent to Participate}

Not applicable.

\section{Consent for Publication}

Not applicable.

\section{Competing Interests}

The authors declare that they have no competing interests.

\section{Received: 19 February 2020 Accepted: 20 November 2020} Published online: 07 December 2020

\section{References}

Aida M, Ishida T, Fukaki H, Fujisawa H, Tasaka M (1997) Genes involved in organ separation in Arabidopsis: an analysis of the cup-shaped cotyledon mutant. Plant Cell 9:841-857

Chen J, Liu Y, Ni J, Wang Y, Bai Y, Shi J, Gan J, Wu Z, Wu P (2011) OsPHF1 regulates the plasma membrane localization of low- and high-affinity inorganic phosphate transporters and determines inorganic phosphate uptake and translocation in rice. Plant Physiol 157:269-278

Chen M, Chen J, Fang J, Guo Z, Lu SJPCT, Culture O (2014) Down-regulation of Sadenosylmethionine decarboxylase genes results in reduced plant length, pollen viability, and abiotic stress tolerance. Plant Cell Tissue Organ Cult 116: 311-322

Dai X, Xu Y, Ma Q, Xu W, Wang T, Xue Y, Chong K (2007) Overexpression of an R1R2R3 MYB gene, OsMYB3R-2, increases tolerance to freezing, drought, and salt stress in transgenic Arabidopsis. Plant Physiol 143:1739-1751

Fujita M, Fujita Y, Maruyama K, Seki M, Hiratsu K, Ohme-Takagi M, Tran LS, Yamaguchi-Shinozaki K, Shinozaki K (2004) A dehydration-induced NAC protein, RD26, is involved in a novel ABA-dependent stress-signaling pathway. Plant J 39:863-876

Gao F, Xiong AS, Peng RH, Jin XF, Xu J, Zhu B, Chen JM, Yao QH (2010) OsNAC52, a rice NAC transcription factor, potentially responds to $A B A$ and confers drought tolerance in transgenic plants. Plant Cell Tissue Org Cult 100:255-262

Hong Y, Zhang H, Huang L, Li D, Song F (2016) Overexpression of a stressresponsive NAC transcription factor gene ONAC022 improves drought and salt tolerance in rice. Front Plant Sci 7:4

Hu H, Dai M, Yao J, Xiao B, Li X, Zhang Q, Xiong L (2006) Overexpressing a NAM, ATAF, and CUC (NAC) transcription factor enhances drought resistance and salt tolerance in rice. Proc Natl Acad Sci U S A 103:12987-12992

Hu H, You J, Fang Y, Zhu X, Qi Z, Xiong L (2008) Characterization of transcription factor gene SNAC2 conferring cold and salt tolerance in rice. Plant Mol Biol 67:169-181
Huh SU, Lee SB, Kim HH, Paek KH (2012) ATAF2, a NAC transcription factor, binds to the promoter and regulates NIT2 gene expression involved in auxin biosynthesis. Mol Cells 34:305-313

Ismail AM, Horie T (2017) Genomics, physiology, and molecular breeding approaches for improving salt tolerance. Annu Rev Plant Biol 68:405-434

Jeong JS, Kim YS, Baek KH, Jung H, Ha SH, Do Choi Y, Kim M, Reuzeau C, Kim JK (2010) Root-specific expression of OsNAC10 improves drought tolerance and grain yield in rice under field drought conditions. Plant Physiol 153:185-197

Jeong JS, Kim YS, Redillas MC, Jang G, Jung H, Bang SW, Choi YD, Ha SH, Reuzeau C, Kim JK (2013) OsNAC5 overexpression enlarges root diameter in rice plants leading to enhanced drought tolerance and increased grain yield in the field. Plant Biotechnol J 11:101-114

Jiang D, Zhou L, Chen W, Ye N, Xia J, Zhuang C (2019) Overexpression of a microRNA-targeted NAC transcription factor improves drought and salt tolerance in Rice via ABA-mediated pathways. Rice (N Y) 12:76

Kim S, Kang JY, Cho DI, Park JH, Kim SY (2004) ABF2, an ABRE-binding bZIP factor, is an essential component of glucose signaling and its overexpression affects multiple stress tolerance. Plant J 40:75-87

Liu Q, Kasuga M, Sakuma Y, Abe H, Miura S, Yamaguchi-Shinozaki K, Shinozaki K (1998) Two transcription factors, DREB1 and DREB2, with an EREBP/AP2 DNA binding domain separate two cellular signal transduction pathways in drought- and low-temperature-responsive gene expression, respectively, in Arabidopsis. Plant Cell 10:1391-1406

Ma X, Zhang Q, Zhu Q, Liu W, Chen Y, Qiu R, Wang B, Yang Z, Li H, Lin Y, Xie Y, Shen R, Chen S, Wang Z, Chen Y, Guo J, Chen L, Zhao X, Dong Z, Liu YG (2015) A robust CRISPR/Cas9 system for convenient, high-efficiency multiplex genome editing in monocot and dicot plants. Mol Plant 8:1274-1284

Mao C, Lu S, Lv B, Zhang B, Shen J, He J, Luo L, Xi D, Chen X, Ming F (2017) A rice NAC transcription factor promotes leaf senescence via $A B A$ biosynthesis. Plant Physiol 174:1747-1763

Mishra M, Kanwar P, Singh A, Pandey A, Kapoor S, Pandey GK (2013) Plant omics: genome-wide analysis of ABA repressor1 (ABR1) related genes in rice during abiotic stress and development. OMICS 17:439-450

Nakashima K, Tran LS, Van Nguyen D, Fujita M, Maruyama K, Todaka D, Ito Y, Hayashi N, Shinozaki K, Yamaguchi-Shinozaki K (2007) Functional analysis of a NAC-type transcription factor OsNAC6 involved in abiotic and biotic stressresponsive gene expression in rice. Plant J 51:617-630

Nambara E, Marion-Poll A (2005) Abscisic acid biosynthesis and catabolism. Annu Rev Plant Biol 56:165-185

Nuruzzaman M, Manimekalai R, Sharoni AM, Satoh K, Kondoh H, Ooka H, Kikuchi S (2010) Genome-wide analysis of NAC transcription factor family in rice. Gene 465:30-44

Ouyang SQ, Liu YF, Liu P, Lei G, He SJ, Ma B, Zhang WK, Zhang JS, Chen SY (2010) Receptor-like kinase OsSIK1 improves drought and salt stress tolerance in rice (Oryza sativa) plants. Plant J 62:316-329

Puranik S, Sahu PP, Srivastava PS, Prasad M (2012) NAC proteins: regulation and role in stress tolerance. Trends Plant Sci 17:369-381

Riechmann JL, Meyerowitz EM (1998) The AP2/EREBP family of plant transcription factors. Biol Chem 379:633-646

Sachs MM, Ho THD (1986) Alteration of gene expression during environmental stress in plants. Annu Rev Plant Physiol 37:363-376

Sakuraba Y, Piao W, Lim JH, Han SH, Kim YS, An G, Paek NC (2015) Rice ONAC106 inhibits leaf senescence and increases salt tolerance and tiller angle. Plant Cell Physiol 56:2325-2339

Serra TS, Figueiredo DD, Cordeiro AM, Almeida DM, Lourenço T, Abreu IA, Sebastián A, Fernandes L, Contreras-Moreira B, Oliveira MM, Saibo NJ (2013) OsRMC, a negative regulator of salt stress response in rice, is regulated by two AP2/ERF transcription factors. Plant Mol Biol 82:439-455

Shim JS, Oh N, Chung PJ, Kim YS, Choi YD, Kim JK (2018) Overexpression of OsNAC14 improves drought tolerance in rice. Front Plant Sci 9:310

Souer E, van Houwelingen A, Kloos D, Mol J, Koes R (1996) The no apical meristem gene of Petunia is required for pattern formation in embryos and flowers and is expressed at meristem and primordia boundaries. Cell 85:159-170

Sperotto RA, Ricachenevsky FK, Duarte GL, Boff T, Lopes KL, Sperb ER, Grusak MA, Fett JP (2009) Identification of up-regulated genes in flag leaves during rice grain filling and characterization of OsNAC5, a new ABA-dependent transcription factor. Planta 230:985-1002

Takasaki H, Maruyama K, Kidokoro S, Ito Y, Fujita Y, Shinozaki K, YamaguchiShinozaki K, Nakashima K (2010) The abiotic stress-responsive NAC-type 
transcription factor OsNAC5 regulates stress-inducible genes and stress tolerance in rice. Mol Gen Genomics 284:173-183

Tang N, Zhang H, Li X, Xiao J, Xiong L (2012) Constitutive activation of transcription factor OsbZIP46 improves drought tolerance in rice. Plant Physiol 158:1755-1768

VanWallendael A, Soltani A, Emery NC, Peixoto MM, Olsen J, Lowry DB (2019) A molecular view of plant local adaptation: incorporating stress-response networks. Annu Rev Plant Biol 70:559-583

Wang Q, Guan Y, Wu Y, Chen H, Chen F, Chu C (2008) Overexpression of a rice OsDREB1F gene increases salt, drought, and low temperature tolerance in both Arabidopsis and rice. Plant Mol Biol 67:589-602

Wei K, Chen H (2018) Global identification, structural analysis and expression characterization of cytochrome P450 monooxygenase superfamily in rice. BMC Genomics 19:35

Xie Q, Frugis G, Colgan D, Chua NH (2000) Arabidopsis NAC1 transduces auxin signal downstream of TIR1 to promote lateral root development. Genes Dev 14:3024-3036

Yamaji N, Ma JF (2007) Spatial distribution and temporal variation of the rice silicon transporter Lsi1. Plant Physiol 143:1306-1313

Yang S, Xu K, Chen S, Li T, Xia H, Chen L, Liu H, Luo L (2019) A stress-responsive bZIP transcription factor OsbZIP62 improves drought and oxidative tolerance in rice. BMC Plant Biol 19:260

Yao L, Cheng X, Gu Z, Huang W, Li S, Wang L, Wang Y-F, Xu P, Ma H, Ge X (2018) The AWPM-19 family protein OsPM1 mediates abscisic acid influx and drought response in rice. Plant Cell 30:1258

Yu SW, Huang AN, Li J, Gao L, Feng YN, Pemberton E, Chen CL (2018) OsNAC45 plays complex roles by mediating POD activity and the expression of development-related genes under various abiotic stresses in rice root. Plant Growth Regul 84:519-531

Yuan X, Wang H, Cai J, Bi Y, Li D, Song F (2019) Rice NAC transcription factor ONACO66 functions as a positive regulator of drought and oxidative stress response. BMC Plant Biol 19:278

Zheng X, Chen B, Lu G, Han B (2009) Overexpression of a NAC transcription factor enhances rice drought and salt tolerance. Biochem Biophys Res Commun 379:985-989

Zhu JK (2002) Salt and drought stress signal transduction in plants. Annu Rev Plant Biol 53:247-273

\section{Publisher's Note}

Springer Nature remains neutral with regard to jurisdictional claims in published maps and institutional affiliations.

\section{Submit your manuscript to a SpringerOpen ${ }^{\circ}$ journal and benefit from:}

- Convenient online submission

- Rigorous peer review

- Open access: articles freely available online

- High visibility within the field

- Retaining the copyright to your article

Submit your next manuscript at $\boldsymbol{\nabla}$ springeropen.com 